\title{
An uncommon case of random fire-setting behavior associated with Todd paralysis: A case report
}

Masayuki Kanehisa', Katsuhiko Morinaga', Hisae Kohno', Yoshihiro Maruyama', Taiga Ninomiya', Yoshinobu Ishitobi', Yoshihiro Tanaka', Jusen Tsuru', Hiroaki Hanada', Tomoya Yoshikawa² and Jotaro Akiyoshi ${ }^{{ }^{*}}$

\begin{abstract}
Background: The association between fire-setting behavior and psychiatric or medical disorders remains poorly understood. Although a link between fire-setting behavior and various organic brain disorders has been established, associations between fire setting and focal brain lesions have not yet been reported. Here, we describe the case of a 24-year-old first time arsonist who suffered Todd's paralysis prior to the onset of a bizarre and random fire-setting behavior.
\end{abstract}

Case presentation: A case of a 24-year-old man with a sudden onset of a bizarre and random fire-setting behavior is reported. The man, who had been arrested on felony arson charges, complained of difficulties concentrating and of recent memory disturbances with leg weakness. A video-EEG recording demonstrated a close relationship between the focal motor impairment and a clear-cut epileptic ictal discharge involving the bilateral motor cortical areas. The SPECT result was statistically analyzed by comparing with standard SPECT images obtained from our institute (easy Z-score imaging system; eZIS). eZIS revealed hypoperfusion in cingulate cortex, basal ganglia and hyperperfusion in frontal cortex,. A neuropsychological test battery revealed lower than normal scores for executive function, attention, and memory, consistent with frontal lobe dysfunction.

Conclusion: The fire-setting behavior and Todd's paralysis, together with an unremarkable performance on tests measuring executive function fifteen months prior, suggested a causal relationship between this organic brain lesion and the fire-setting behavior. The case describes a rare and as yet unreported association between random, impulse-driven fire-setting behavior and damage to the brain and suggests a disconnection of frontal lobe structures as a possible pathogenic mechanism.

Keywords: Fire setting, Arson, Lacunar stroke, Frontal lobe dysfunction, Focal epilepsy, Prolonged seizures, Ictal paralysis, Todd's paresis

\section{Background}

The association between fire-setting behavior and psychiatric or medical disorders remains poorly understood. Thus far, studies on the characteristics of firesetters have revealed associations with substance use disorders, personality disorders, mental retardation, schizophrenia-, mood-, and neurotic-spectrum disorders, and impulse-control disorders, including pyromania [1].

\footnotetext{
* Correspondence: akiyoshi@oita-u.ac.jp

'Department of Neuropsychiatry, Oita University Faculty of Medicine, Oita, Hasama-Machi 879-5593, Japan

Full list of author information is available at the end of the article
}

Rare associations of fire-setting behavior with sexual disorders (e.g. paraphilias), luteal phase dysphoric disorder, Asperger's syndrome, delirious states, dementia, epilepsy, Klinefelter syndrome, XYY karyotype, Kleine-Levin syndrome, Fahr disease, and mutations in the gene encoding for monoamine oxidase A have also been reported [1-5]. Although a link between fire-setting behavior and various organic brain disorders has been established [6], associations between fire setting and focal brain lesions have not yet been reported. Transient paresis of an arm or leg following an epileptic seizure is probably the most classic lateralizing postictal paresis (PP) [7]. Here, we

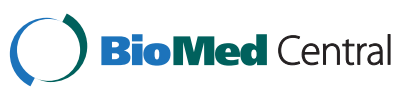


describe the case of a 24-year-old first time arsonist who suffered Todd's paralysis prior to the onset of a bizarre and random fire-setting behavior.

\section{Case presentation}

A 24-year-old, right-handed Japanese man with a ninthgrade education was referred to us by the court for pretrial evaluation. His sister, aunt and cousin have been suffering from epilepsy. He had no history of antisocial behavior. Fifteen months ago, he suffered from headache, nausea, vomiting, general malaise, abdominal discomfort, vertigo, and tinnitus during a shopping experience and could not maintain an upright stance or walk. He was transported to the hospital, and was diagnosed with vertigo and generalized anxiety disorder. Fourteen months ago he showed the same symptoms, as well as a loss of consciousness and numbness in the legs during a lunch break. Thirteen months ago, he stopped driving because he complained about headaches and vertigo, although he could move his legs. He could not remember this episode. Eight months ago a psychiatrist diagnosed epilepsy with an electroencephalogram (EEG) and started to administer clonazepam (1 mg). After this he spent most of his days fishing without a job.

The suspect had been arrested on felony arson charges after starting at least five separate fires in residential neighborhoods in less than a month. The suspect, who had no prior arrests, indictments, or convictions, confessed to setting fires indiscriminately and seemingly without purpose to a bamboo broom, styrofoam, a towel, and a wooden board near a junior high school, a warehouse, a boat, and a shrine, respectively. When he set fire to some items he felt vertigo and could not maintain power in the legs.

The pre-trial evaluation involved the assessment of (i) the examinee's current mental state, (ii) the examinee's criminal responsibility, primarily depending on the examinee's mental state at the time of the offence, (iii) the examinee's risk for criminal recidivism, and (iv) the suitability of a court order for mental health treatment to reduce criminal recidivism.

Upon presentation, the examinee appeared to feel well but complained of difficulties concentrating and of a recent impairment in memory. He claimed to have had no intent to violate the law and appeared to have no explanation for his fire-setting behavior. He recalled waking up in the early morning hours and leaving his home to roam the residential neighborhood. He also recalled feeling somehow strange before setting fire to various items haphazardly. The examinee denied any sexual fantasies or arousal associated with the fire-setting behavior.

\section{Medical history}

The examinee was taking no regular medication but had a history of alcohol abuse since his adolescent years.
Alcohol intoxication was therefore considered as a possible cause for the fire-setting behavior but was later dismissed as no evidence for recent alcohol consumption emerged.

\section{Mental status examination}

Initial multiaxial diagnostic assessment according to classifications provided by the Fourth Edition of the Diagnostic and Statistical Manual of Mental Disorders-Text Revision (DSM-IV-TR, American Psychiatric Association, Washington DC, 2000) revealed mild mental retardation. There was no evidence for delusions, hallucinations, manic episodes, or possible axis II disorders relevant to the fire-setting behavior. Axis III evaluation revealed a history of Todd's paralysis, which raised the possibility of a delirious state as a possible cause for the observed firesetting behavior [4]. Axis IV evaluation did not reveal burdening psychosocial and environmental factors including occupational and housing problems. Further investigations showed that, prior to the fire-setting, he was unemployed for eight months after a security guard job. Finally, axis V assessment of global functioning revealed a score below 65 (maximum score 100).

\section{Laboratory data}

Laboratory results were normal or borderline pathologic. A diminished lymphocyte count $(990 / \mathrm{ml})$, a slightly elevated C-reactive protein $(2.3 \mathrm{mg} / \mathrm{l})$, and marginally diminished $\mathrm{Ca}^{++}(10.4 \mathrm{mg} / \mathrm{l})$ were found. Serologic testing showed no evidence for infection with Treponemapallidum, Borreliaburgdorferi, hepatitis B or C viruses, or HIV $1 / 2$.

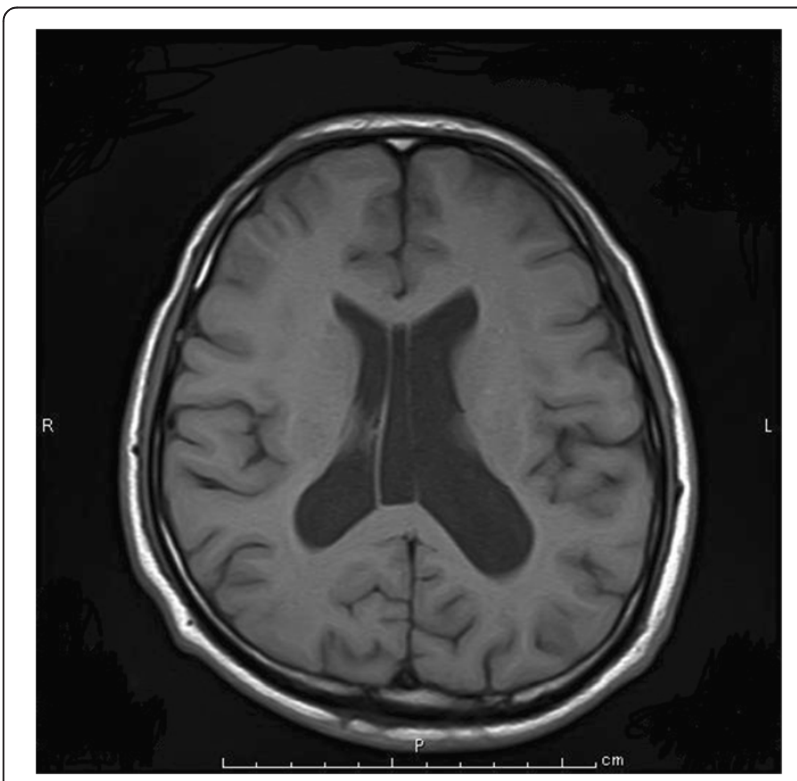

Figure $1 \mathrm{MRI}$ of the brain and dilated lateral ventricle. 


\section{Neuroimaging data}

Cerebral magnetic resonance imaging (MRI) revealed atrophy of the brain and dilated lateral ventricle (Figure 1). An easy Z-score imaging system (eZIS) program was used to analyze perfusion single-photon emission computed tomography (SPECT) images of the study participants [8-10]. SPECT images obtained from each participant were subjected to anatomic standardization using statistical parametric mapping two with an original Tc-99 m ECD template followed by isotropic 12- $\mathrm{mm}$ smoothing and compared with the mean and standard deviation (SD) of SPECT images obtained from a normal database that had been previously established at our institute. The SPECT result was statistically analyzed by comparing with standard SPECT images obtained from our institute (easy Z-score imaging system; eZIS). eZIS revealed hyperperfusion in frontal cortex (Figure 2). eZIS from ictal - interictal ECD-SPECT showed hyperperfusion in the frontal cortex (Figure 3). eZIS from interictal - ictal Tc-99 m ECD-SPECT showed hypoperfusion in cingulate cortex, basal ganglia (Figure 4).

\section{Neurologic examination}

Bilateral PP of the bilateral legs (motor deficits without sensory deficits) lasted approximately 5 minutes. He was confused during ictal events, and suffered from headache, and retrograde and anterograde amnesia after ictal events.

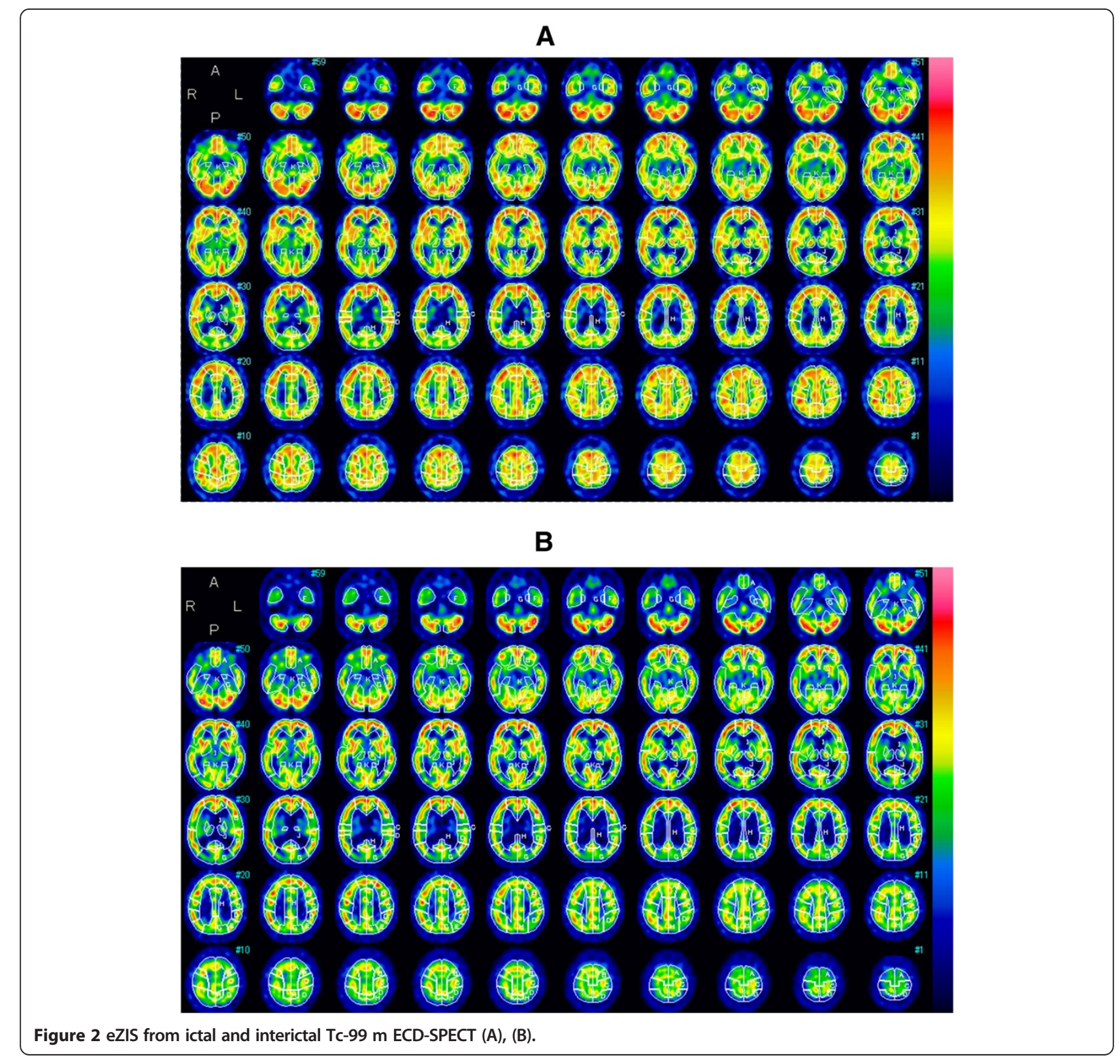




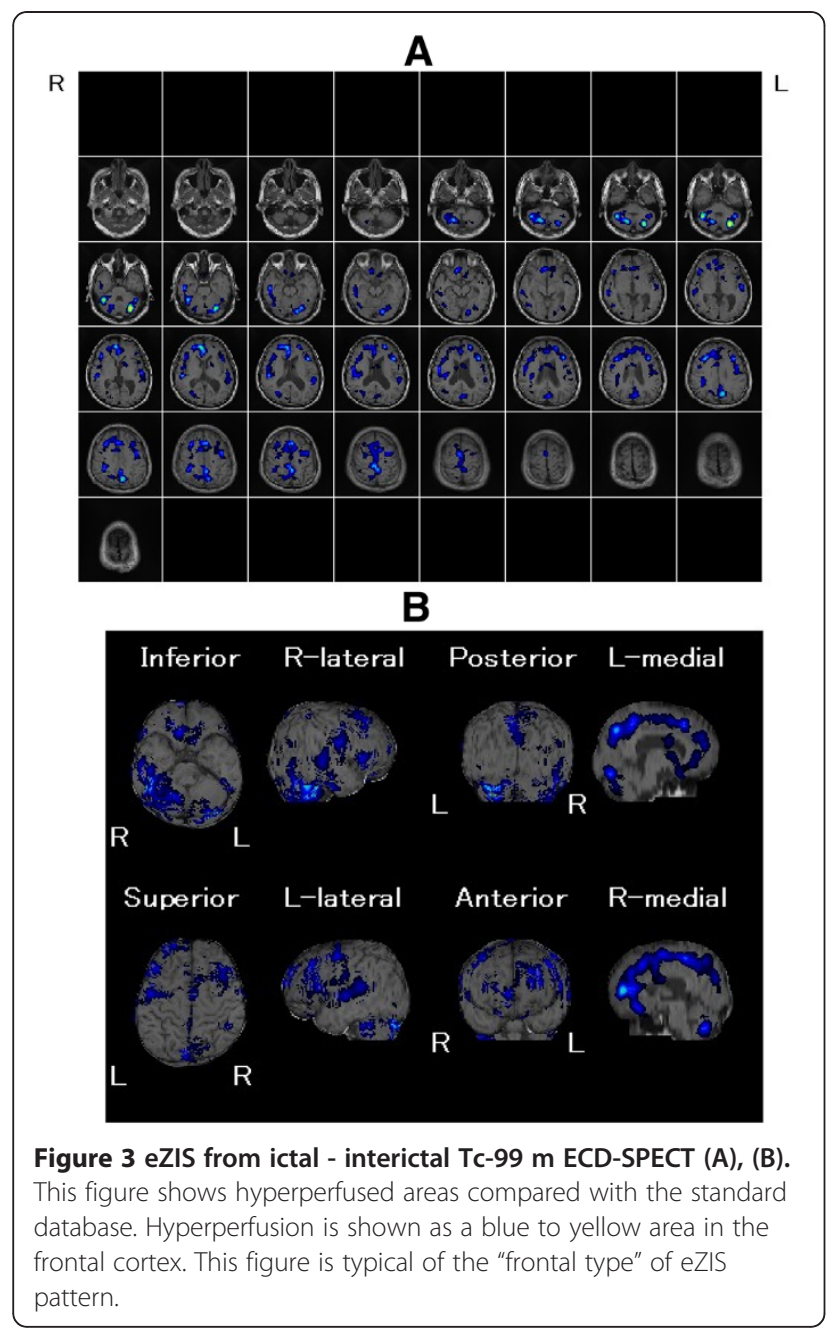

\section{Other diagnostic procedures}

Further examination showed that an electrocardiogram was normal. A video-EEG was performed. The tracing just before epilepsy was characterized by the presence of irregular slow waves (Figure 5A). The tracing during epilepsy was characterized by the presence of an ictal discharge of repetitive spikes localized in the frontal, central, and parietal regions. The appearance of the motor deficit (starting from the hand) was accompanied by a clear-cut ictal discharge of repetitive spikes involving the right frontal, central, and parietal regions (Figure 5B). The discharge clearly correlated with the severity of the neurologic deficit, being more sustained when the paralysis was complete and more irregular and fragmented when it was less severe.

\section{Neuropsychological testing}

We performed a number of neuropsychological tests with particular emphasis on the assessment of executive function and control [11]. Performance on the n-back

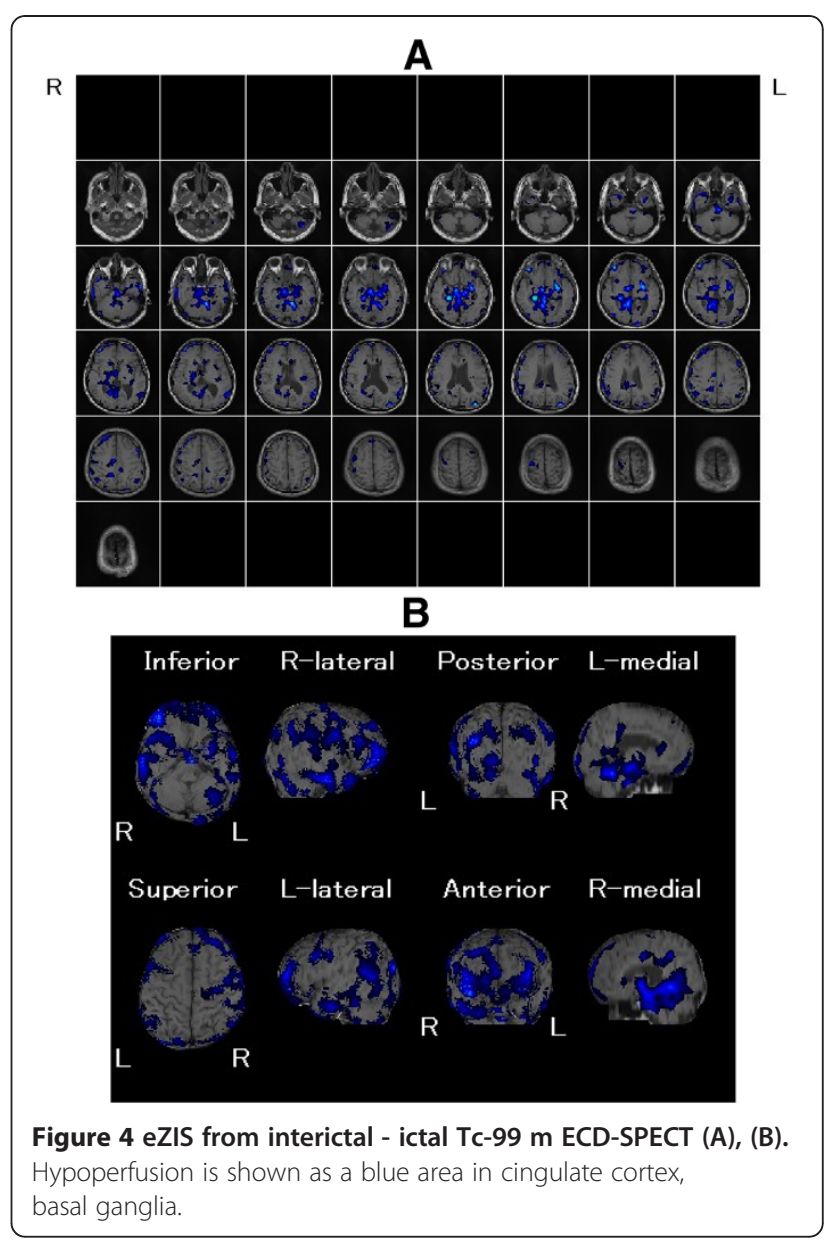

task, which is sensitive to frontal lobe damage [12], was poor (trials correct; 1-back: 75\%, 2-back: 42\%, 3-back: $16 \%)$. Assessment of letter-based word retrieval using the phonemic version of the Thurstone Word Fluency Test yielded remarkably poor results ( 21 words produced in $3 \mathrm{~min}$ ), consistent with a loss-of-function of the left frontal lobe [13]. With respect to the Wisconsin Card Sorting Test (WCST), the error rate, the number of categories achieved, and the number of perseverative errors of the Nelson type were $52.6 \%, 4$, and 20 , respectively. The Wechsler Adult Intelligence Scale-III (WAIS-III) revealed scores below the normal range (scaled score $10.1 \pm 0.9$ ) for three performance subtests: arithmetic (score 3), picture completion (score 1), digit symbol coding (score 12), and symbol search (score 1). These scores indicated a moderate impairment in perceptual organization and a severe impairment in processing speed, consistent with the involvement of frontal brain regions in these tasks [14]. An important finding across all neuropsychological tests was the examinee's unawareness of his test performance.

Most likely, these cognitive impairments developed as a result of epilepsy. In particular, performance on the 


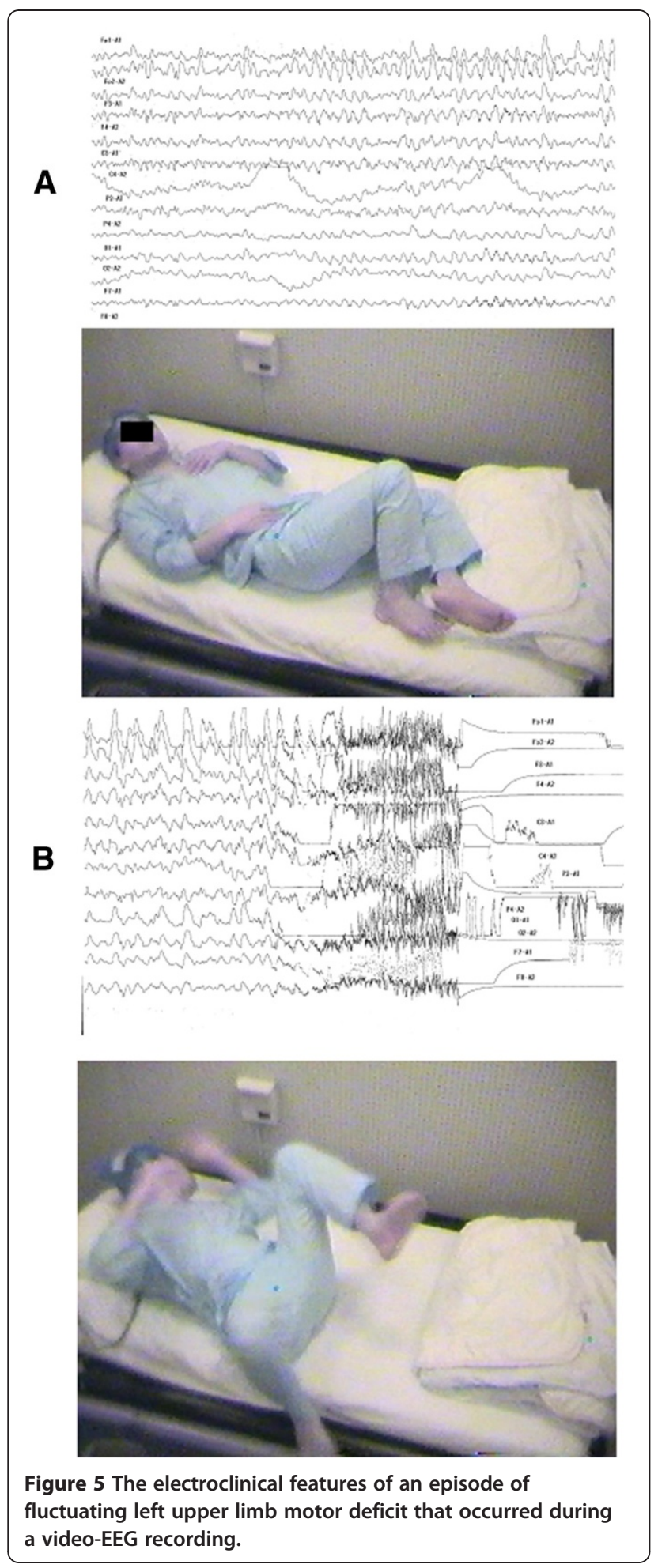

three WAIS-III subtests with low scores was normal in the pre-stroke condition: picture completion (score 8), block design (score 7), and digit symbol coding (score 8 ). The average premorbid intelligence quotient (IQ) score, obtained at the same time, was 63 (performance score 68; verbal score 64). Treatment with carbamazepine was started and led to complete seizure control.

\section{Discussion}

Arson is frequently associated with psychiatric morbidity and with previous non-violent or violent offences [15]. In contrast, our case suggests an association between an isolated, first-time arson offence and Todd's paralysis. Todd's paralysis describes focal weakness in a part of the body after a seizure. Our case showed bilateral leg weakness, and this weakness recovered completely within 48 hours. The precise mechanism that links damage of the motor cortex to this complex, impulse-driven behavior is not known. Neuropsychological and neuropsychiatric findings suggest temporal and frontal lobe dysfunctions in violent offenders and these dysfunctions appear to be more pronounced in the dominant hemisphere [16].

However, lesions in the hypoperfused regions within the frontal cortex and basal ganglia might result in a disconnection and deactivation of the frontal cortex. Consistent with this concept are more recent observations linking cognitive inflexibility with prefrontal depletion of serotonin [17]. Moreover, and in agreement with our findings, neuropsychological deficits (including memory impairment) have been associated with hypoperfusion within the frontal cortex and basal ganglia $[18,19]$. Finally, a neural framework controlling impulsive behavior, decision-making, and willpower has been proposed incorporating components of this framework, and is primarily localized to frontal lobe structures [20].

Here we argue that, in our case, disconnection of the frontal lobe resulted in impairments of cognition, primarily executive functions involving planning and judgment, as well as partial loss of memory and impulse control functions. Together, this led to a bizarre type of impulse-driven, non-intentional fire-setting behavior. Consequently, the examinee's capacity to form a specific intent at the time of the offence remained uncertain, implying lack of capacity to form mensrea [21], which, at least in part, negated criminal responsibility.

Based on our pre-trial evaluation, a diagnosis of personality change (disinhibited behavior) due to brain damage and dysfunction (DSM-IV-TR: 310.1) was made, combined with anosognosic features (i.e., the suspect's unawareness of his impaired cognitive functions). The examinee was found not criminally responsible. However, the examinee was deemed at risk for criminal recidivism as no specific treatment for his condition existed. We recommended continuing prophylactic treatment with antiepileptic medication to help prevent further hypoxic-ischemic insults. The case was closed with a verdict of guilty by reason of legal sanity and the examinee was transferred to a prison. Neuropsychological 
reassessment may help estimate the examinee's risk for future criminal recidivism.

\section{Conclusion}

The fire-setting behavior and Todd's paralysis, together with an unremarkable performance on tests measuring executive function fifteen months prior, suggested a causal relationship between this organic brain lesion and the fire-setting behavior. The case describes a rare and as yet unreported association between random, impulsedriven fire-setting behavior and damage to the brain and suggests a disconnection of frontal lobe structures as a possible pathogenic mechanism.

\section{Abbreviations}

DSM-IV-TR, The Fourth Edition of the Diagnostic and Statistical Manual of Mental Disorders, Text Revision; EEG, Electroencephalogram; eZIS, Z-score imaging system; MRI, Magnetic resonance imaging; WAIS-III, The Wechsler Adult Intelligence Scale-III; SPECT, Single-photon emission computed tomography.

\section{Competing interests}

Authors have no competing interests to declare that are relevant to the content of this submission.

\section{Authors' contributions}

MK and KM followed up the patient during the admission. YM and TN participated in EEG study. YI and YT examined SPECT study, HK, JT and HH examined psychological test examination, JA planed the study, and participated in its design and coordination and helped to draft the manuscript. All authors read and approved the final manuscript.

\section{Acknowledgements}

Written consent was obtained from their relative for publication of study.

\section{Author details}

${ }^{1}$ Department of Neuropsychiatry, Oita University Faculty of Medicine, Oita, Hasama-Machi 879-5593, Japan. FUJIFILM RI Pharma Co., Ltd., Tokyo

104-0031, Japan.

Received: 7 October 2011 Accepted: 23 May 2012

Published: 31 August 2012

\section{References}

1. Geller JL: Pathological firesetting in adults. Int J Law Psychiatry 1992, 15(3):283-302

2. Powers PS, Gunderman R: Kleine-Levin syndrome associated with fire setting. Am J Dis Child 1978, 132(8):786-789.

3. Brunner $\mathrm{HG}$, Nelen $\mathrm{M}$, Breakefield $\mathrm{XO}$, Ropers $\mathrm{HH}$, van Oost BA: Abnormal behavior associated with a point mutation in the structural gene for monoamine oxidase A. Science 1993, 262(5133):578-580.

4. Maharajh $\mathrm{HD}$, Konings $\mathrm{M}$ : Fire setting in a patient with hyperglycaemic delirium. J Forensic Sci 2006, 51(4):940.

5. Shirahama M, Akiyoshi J, Ishitobi $Y$, Tanaka $Y$, Tsuru J, Matsushita H, Hanada $\mathrm{H}$, Kodama $\mathrm{K}$ : A young woman with visual hallucinations, delusions of persecution and a history of performing arson with possible three-generation Fahr disease. Acta PsychiatrScand 2010, 121(1):75-77.

6. Puri BK, Baxter R, Cordess CC: Characteristics of fire-setters. A study and proposed multiaxial psychiatric classification. Br J Psychiatry 2005, 166(3):393-396.

7. Todd RB: Clinical lectures on paralysis, disease of the brain and other affection of the nervous system. London: Univ. of London; 1854.

8. Minoshima S, Frey KA, Koeppe RA, Foster NL, Kuhl DE: A diagnostic approach in Alzheimer's disease using three-dimensional stereotactic surface projections of fluorine-18-FDG PET. J NuCl Med 1995, 36(7):1238-1248.
9. Matsuda H: Role of neuroimaging in Alzheimer's disease, with emphasis on brain perfusion SPECT. J NuCl Med 2007, 48(8):1289-1300.

10. Matsuda H, Mizumura S, Nagao T, Ota T, lizuka T, Nemoto K, Takemura N, Arai $\mathrm{H}$, Homma A: Automated discrimination between very early Alzheimer disease and controls using an easy Z-score imaging system for multicenter brain perfusion single-photon emission tomography. AJNR Am J Neuroradiol 2007, 28(4):731-736.

11. Strauss E, Sherman EMS: A Compendium of Neuropsychological Tests: Administration, Norms, and Commentary. New York: Oxford University Press; 2006.

12. Kirchner WK: Age differences in short-term retention of rapidly changing information. J ExpPsychol 1958, 55(4):352-358.

13. Baldo JV, Shimamura AP, Delis DC, Kramer J, Kaplan E: Verbal and design fluency in patients with frontal lobe lesions. J IntNeuropsycholSoc 2001, 7(5):586-596.

14. Gläscher J, Tranel D, Paul LK, Rudrauf D, Rorden C, Hornaday A, Grabowski T, Damasio $H$, Adolphs $R$ : Lesion mapping of cognitive abilities linked to intelligence. Neuron 2009, 61(5):681-691.

15. Repo E, Virkkunen M, Rawlings R, Linnoila M: Criminal and psychiatric histories of Finnish arsonists. Acta PsychiatrScand 1997, 95(4):318-323.

16. Volavka J, Martell D, Convit A: Psychobiology of the violent offender. J Forensic Sci 1992, 37(1):237-251.

17. Clarke HF, Dalley JW, Crofts HS, Robbins TW, Roberts AC: Cognitive inflexibility after prefrontal serotonin depletion. Science 2004, 304(5672):878-880.

18. Chukwudelunzu FE, Meschia JF, Graff-Radford NR, Lucas JA: Extensive metabolic and neuropsychological abnormalities associated with discrete infarction of the genu of the internal capsule. J Neurol Neurosurg Psychiatry 2001, 71(5):658-662.

19. Tatemichi TK, Desmond DW, Prohovnik I, Cross DT, Gropen TI, Mohr JP Stern Y: Confusion and memory loss from capsular genu infarction: a thalamocortical disconnection syndrome? Neurology 1992, 42(10):1966-1979.

20. Bechara A: Decision making, impulse control and loss of willpower to resist drugs: a neurocognitive perspective. Nat Neurosci 2005, 8(11):1458-1463.

21. Barratt ES, Felthous AR: Impulsive versus premeditated aggression: implications for mensrea decisions. BehavSci Law 2003, 21(5):619-630.

doi:10.1186/1471-244X-12-132

Cite this article as: Kanehisa et al: An uncommon case of random fire-setting behavior associated with Todd paralysis: A case report. BMC Psychiatry 2012 12:132.

\section{Submit your next manuscript to BioMed Central and take full advantage of:}

- Convenient online submission

- Thorough peer review

- No space constraints or color figure charges

- Immediate publication on acceptance

- Inclusion in PubMed, CAS, Scopus and Google Scholar

- Research which is freely available for redistribution 